\title{
面向可持续发展的二氧化碳化学
}

何良年

南开大学化学学院, 元素有机化学国家重点实验室, 天津 300071

E-mail: heln@nankai.edu.cn

\section{Carbon dioxide chemistry towards sustainable development}

\section{Liang-Nian He}

State Key Laboratory of Elemento-Organic Chemistry, College of Chemistry, Nankai University, Tianjin 300071, China E-mail: heln@nankai.edu.cn

doi: 10.1360/TB-2020-1119

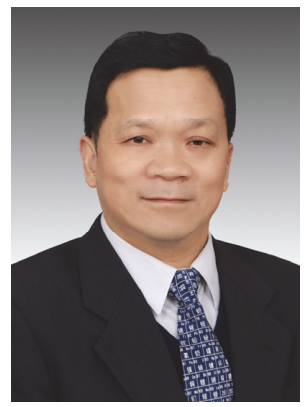

\section{何良年}

南开大学教授, 英国皇家 化学会士(FRSC). 从事 绿色化学、二氧化碳资源 化利用、生物质高值化等 领域的研究工作。在二氧 化碳化学及可再生碳基 能源化学领域取得了系 列创新成果, 编著了《二 氧化碳化学》与《绿色化 学基本原理》, 推动了二 氧化碳化学领域的发展. 曾获天津市自然科学奖、 军队科学技术进步奖.
有效利用资源、保护环境、发展绿色化学已成为大势所趋. 二氧化碳过度排 放是全球变暖、极端天气和荒漠化等全球环境变化的主要诱导因素. 将 $\mathrm{CO}_{2}$ 定向 转化为有价值的化学品、能源与材料, 也可一定程度上缓解环境问题, 并减缓人 类对化石能源的依赖. 将丰富、价廉、可再生的 $\mathrm{CO}_{2}$ “变废为宝、高值化利用”, 以 部分替代化石原料, 满足可持续发展需求。当今世界正处于能源供给从过度依赖 化石能源向基于太阳燃料转型的过渡时期, 利用太阳能以及可再生电能将 $\mathrm{CO}_{2}$ 还 原为生产碳氢燃料, 已经成为本领域有巨大应用潜力及挑战的热点课题. 总之, 面向可持续发展的二氧化碳化学研究具有重要学术价值和广阔的应用前景 ${ }^{[1]}$.

与石化工业相比, 现有 $\mathrm{CO}_{2}$ 基的化工产品种类还不多, 尤其是对映选择性催 化反应少; $\mathrm{CO}_{2}$ 热力学上的低能态, 能量是其转化利用的基础. $\mathrm{CO}_{2}$ 的惰性决定了 活化是进行 $\mathrm{CO}_{2}$ 转化反应前提, 而活化的关键取决于高效催化体系. 关键科学问 题源于 $\mathrm{CO}_{2}$ 活化反应机理还不明确, 难以进行催化剂的理性设计. 因此, 需探讨 催化作用机制, 形成催化剂设计新方法; 突破 $\mathrm{CO}_{2}$ 还原产物精准调控问题, 以实 现 $\mathrm{CO}_{2}$ 定向转化. 最引人注目的是光电催化还原 $\mathrm{CO}_{2}$ 领域, 通过光及可再生电催 化还原, 以克服转化中的能源问题, 开辟利用太阳能光解水制氢与转化 $\mathrm{CO}_{2}$ 相结 合的新途径, 即可再生能源储存和 $\mathrm{CO}_{2}$ 转化的可持续发展模式. 此外, 将 $\mathrm{CO}_{2}$ 转 化为大宗化学品甲醇、甲酸、一氧化碳和苯酚以及高附加值产品如丙烯酸酯、杂 环化合物等, 有利于提高经济性. 从有害试剂替代及原子经济性来看, 作为替代 化石资源的合成方法, 基于 $\mathrm{CO}_{2}$ 的合成路线具有低碳、环境友好的巨大应用潜力.

我国在二氧化碳化学领域的研究与开发队伍规模大, 所取得的成就处于国际先 进水平, 围绕二氧化碳化学与资源化利用领域的基础、应用和前沿问题, 在与 $\mathrm{CO}_{2}$ 转 化反应相关的活化机理、高活性催化剂设计开发、转化新方法、新反应、新途径与 新策略、光、电催化方法、 $\mathrm{CO}_{2}$ 制备化学品/能源产品/聚合物等方面都取得了突破. 因 此, 以“面向可持续发展的二氧化碳化学”为主题的中国化学会首届二氧化碳资源化 利用大会, 于 2019 年 11 月 8 10 日在南开大学成功召开. 在中国化学会绿色化学专 业委员会及韩布兴院士支持下，组织基于本次大会成果的“面向可持续发展的二氧化 碳化学”专题, 以展现我国学者在该领域的研究成果, 促进学术交流, 发挥我国在有 
机化学、精准合成、催化材料、绿色介质及高分子合成等研究领域的学科优势, 推动二氧化碳化学研究. 本专 题侧重于收录二氧化碳化学与资源化利用领域, 包括二氧化碳化学原理与应用(催化活化与催化剂设计)、二氧 化碳定向转化的新反应/新途径/新方法、光电催化方法、 $\mathrm{CO}_{2}$ 制备化学品/ $\mathrm{CO}_{2}$ 基聚合物以及捕集与分离等方面 所取得的最新研究成果与进展评述; 并分析与探讨 $\mathrm{CO}_{2}$ 领域的科学问题与技术难题、所面临的机遇、挑战和 未来发展方向.

本专题共收录 17 篇论文, 分两期刊出. 本期包含 1 篇进展、4 篇评述和 3 篇研究论文. 孙建敏课题组 ${ }^{[2]}$ 围 绕 $\mathrm{CO}_{2}$ 与邻氨基苯腈羒环化合成咺唑啉- $2,4(1 \mathrm{H}, 3 \mathrm{H})$-二酮反应, 系统综述了不同类型催化剂设计、制备方法及催 化性能等研究进展, 分析了所存在的关键科学问题, 并对适用于 $\mathrm{CO}_{2}$ 转化反应的催化材料进行了展望. 鲁统部 课题组 ${ }^{[3]}$ 对电催化 $\mathrm{CO}_{2}$ 还原制备 $\mathrm{C}_{2}$ 产物进展作了简要评述, 阐明催化剂晶面、尺寸、形貌、担载密度、表面原 子氧化态等对 $\mathrm{C}_{2}$ 产物催化活性和选择性的影响. 李康康、于海、刘恢等人 ${ }^{[4]}$ 针对如何提高酮基催化剂在 $\mathrm{CO}_{2}$ 电 还原为 $\mathrm{C}_{2}$ 的效率以及产物难以定向调控的共性科学问题, 从催化剂本征特性、催化环境与电解装置等方面进 行了分析与总结. 韩宝航课题组 ${ }^{[5]}$ 介绍了多孔聚咔唑的制备方法及其在 $\mathrm{CO}_{2}$ 吸附与催化转化等方面的应用, 分 析了材料结构与性能的关系, 总结归纳了高性能聚合物所需的关键因素. 刘宁课题组 ${ }^{[6]}$ 综述了基于差基、羒基、 氨基、多氢键给体和特殊氢键给体促进的 $\mathrm{CO}_{2}$ 与环氧化物的环加成反应的研究进展, 以期为高效有机催化剂的 设计合成提供一种新思路. 吕小兵棵题组 ${ }^{[7]}$ 以手性 2,3 - 丁二醇为起始原料, 通过迭代合成方法, 采用三光气为羰 化试剂, 制备全同和间同结构的 $\mathrm{CO}_{2}$ /顺式-2,3-环氧丁烷交替共聚物(聚碳酸-2,3-丁烯酯)的四聚体和入聚体, 并 用 ${ }^{13} \mathrm{C}$ 核磁共振谱精确分析其微结构. 王万辉、包明等人 ${ }^{[8]}$ 利用廉价金属铜盐和 $N, N, N^{\prime}, N^{\prime}$-四甲基乙二胺、 $N, N^{\prime}$ 二甲基乙二胺、乙二胺、联嘧啶等配体原位生成金属配合物, 并用于催化硼氢化钠还原 $\mathrm{CO}_{2}$ 制备甲醇, 提出了 可能的催化机理. 宋清文、张㑆等人设设计热力学有利的 $\mathrm{CO}_{2}$ 、炔丙醇和甲醇的三组分串联反应, 成功制备碳酸 二甲酯和 $\alpha$-差基酮，使用廉价锌与有机碱的协同催化体系，对该串联反应进行了系统研究.

值此专题出版之际, 对所有作者、审稿人以及《科学通报》编辑部的大力支持和帮助表示诚挚感谢! 希 望本专题能引起更多关注, 激发年轻学者兴趣, 抛砖引玉, 旨在推动 $\mathrm{CO}_{2}$ 资源化研究与应用及相关学科的进 一步发展 ${ }^{[10]}$, 提升我国在 $\mathrm{CO}_{2}$ 化学理论研究和新技术研发的竞争力和国际地位.

\section{参考文献}

1 Yao X Y, Zhang Y, Gao S, et al. Progress on carbon dioxide chemistry towards sustainable development (in Chinese). J Huazhong Norm Univ Nat Sci, 2019, 53: 834-846 [姚向阳, 张彦, 高嵩, 等. 面向可持续发展的二氧化碳化学研究进展. 华中师范大学学报(自然科学 版), 2019, 53: 834-846]

2 Liu M S, Ping R, Sun J M. Advances in the catalyst development for synthesis of quinazoline-2,4(1H,3H)-diones and their derivatives by chemical fixation of $\mathrm{CO}_{2}$ (in Chinese). Chin Sci Bull, 2020, 65: 3349-3359 [刘猛帅, 平再, 孙建敏. $\mathrm{CO}_{2}$ 催化转化合成喹唑啉2,4(1H,3H)-二酮及其衍生物研究进展. 科学通报, 2020, 65: 3349-3359]

3 Zhang C, Lu T B. Synthesis of $\mathrm{C}_{2}$ products via electrocatalytic $\mathrm{CO}_{2}$ reduction (in Chinese). Chin Sci Bull, 2020, 65: 3401-3417 [张超, 鲁 统部. 电催化二氧化碳还原合成二碳产物. 科学通报, 2020, 65: 3401-3417]

4 Xiang K S, Liu Y C, Yu H, et al. Strategies to improve the performance of copper-based catalyst for electroreduction of $\mathrm{CO}_{2}$ to multicarbon products (in Chinese). Chin Sci Bull, 2020, 65:3360-3372 [向开松, 刘雨程, 于海, 等. 铜基催化剂电还原 $\mathrm{CO}_{2}$ 为多碳产物的提 升策略. 科学通报, 2020, 65: 3360-3372]

5 Wang T X, Han B H. Carbazole-based porous organic polymers for carbon dioxide capture and catalytic conversion (in Chinese). Chin Sci Bull, 2020, 65: 3389-3400 [王天雄, 韩宝航. 多孔聚咔唑用于二氧化碳捕获与催化转化. 科学通报, 2020, 65: 3389-3400]

6 Liu N, Chen F, Tao S. Hydrogen bond donors promoting organocatalyzed cycloaddition of $\mathrm{CO}_{2}$ with epoxides (in Chinese). Chin Sci Bull, 2020, 65: 3373-3388 [刘宁, 陈飞, 陶晟. 氢键给体促进有机催化的 $\mathrm{CO}_{2}$ 与环氧化物的环加成反应. 科学通报, 2020, 65: 3373-3388]

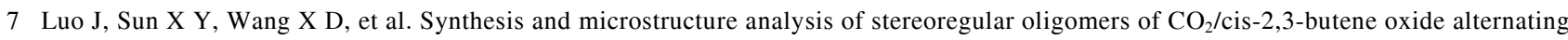
copolymer (in Chinese). Chin Sci Bull, 2020, 65: 3418-3428 [罗婧, 孙星宇, 王霞弟, 等. $\mathrm{CO}_{2} /$ 顺式-2,3-环氧丁烷交替共聚物的立体寡 聚物合成及微结构分析. 科学通报, 2020, 65: 3418-3428]

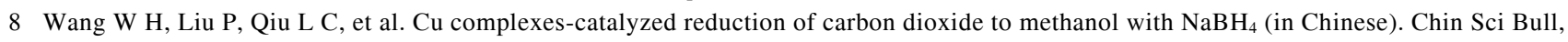
2020, 65: 3438-3445 [王万辉, 刘平, 邱立春, 等. $\mathrm{Cu}$ 配合物催化嗍氢化钠还原 $\mathrm{CO}_{2}$ 制备甲醇. 科学通报, 2020,65: 3438-3445]

9 Zhang Q X, Liu P, Zhang K, et al. Multicomponent cascade strategy of $\mathrm{CO}_{2}$ fixation for synthesis of dimethyl carbonate and $\alpha$-hydroxy ketone (in Chinese). Chin Sci Bull, 2020, 65: 3429-3437 [张乾霞, 刘平, 张㑆，等. 多组分串联策略固定 $\mathrm{CO}_{2}$ 制碳酸二甲酯和 $\alpha$-差基 酮. 科学通报, 2020, 65: 3429-3437]

10 Chen K H, Li H R, He L N. Advance and prospective on $\mathrm{CO}_{2}$ activation and transformation strategy (in Chinese). Chin J Org Chem, 2020, 40: 2195-2207 [陈凯宏, 李红茹, 何良年. 二氧化碳活化和转化策略研究进展. 有机化学, 2020, 40: 2195-2207] 